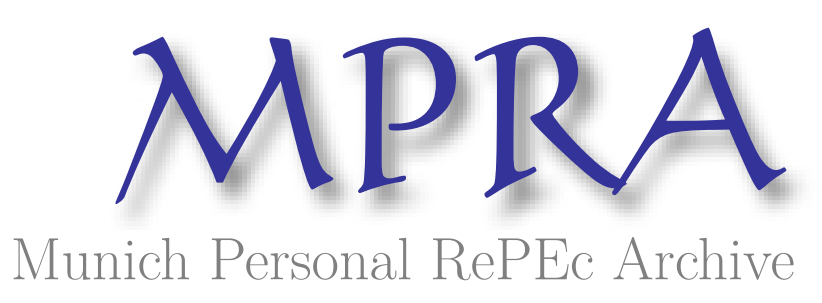

\title{
Modified VCG Mechanisms in \\ Combinatorial Auctions with Budget Constraints
}

Le, Phuong

Stanford University

1 March 2014

Online at https://mpra.ub.uni-muenchen.de/66384/

MPRA Paper No. 66384, posted 01 Sep 2015 06:33 UTC 


\title{
Modified VCG Mechanisms in Combinatorial Auctions with Budget Constraints
}

\author{
Phuong Le ${ }^{1}$ \\ Stanford University
}

\begin{abstract}
I present two modifications of the Vickrey-Clark-Groves mechanism to accommodate bidders' budget constraints in the combinatorial auction setting and show that they are Pareto-Optimal and (partially) incentive compatible in certain domains.
\end{abstract}

Keywords: Vickrey-Clark-Groves, Combinatorial Auctions, Budget Constraints, Pareto-Optimal, Mechanism Design

JEL Classification Code: D44, D47

\section{Introduction}

Bidders' budget constraints have been detected in many auctions (Bulow et al., 2009). The implications of budget constraints in auctions have been examined in various settings. In the single-good setting, Che and Gale $(1996,2000)$ show that all-pay auctions result in higher revenues that first-price sealed-bid auctions and derive an optimal auction (see also Laffront and Robert, 1996). In the multi-unit setting where multiple copies of the same good are sold, Dobzinski et al. (2008) show that when bidders are budget-constrained there is no incentive-compatible Pareto-optimal auction. When budgets are public, they propose the adaptive clinching auction, a modification of the clinching auction in Ausubel (2004), and show that it satisfies Pareto-optimality, individual rationality and incentive compatibility (see also Borgs et al., 2005). In the single divisible good setting, Bhattacharya et al. (2010) show that adaptive clinching makes it sub-optimal to underreport budget, and Hafalir et al. (2011) show that a generalization of the Vickrey auction called the Vickrey with Budgets yields good revenue, incentive and efficiency properties. In the multiple heterogeneous goods setting, budget constraints preclude mechanisms that are Pareto Optimal and incentive compatible (Goel et al., 2012; Dobzinski et al., 2008; Fiat et al., 2011; Lavi and May, 2011). Certain auction designs such as the simultaneous ascending auction (Milgrom, 2000) and the clock auction (Gul and Stacchetti, 2000) implicitly take into account budget constraints by operating through bidders' demands, but are not incentive compatible.

An important question is whether existing mechanisms perform well in the presence of budget constraints, either in their current format or with some modification. This paper studies the Vickrey-ClarkGroves (VCG) mechanism in combinatorial auction setting. A naive application of VCG to the profile of valuations that disregards budget constraints may lead to the outcome where a winning bidder is unable

\footnotetext{
Email address: phuong. le@alumni.stanford.edu (Phuong Le )

URL: https://sites.google.com/site/lp3ides (Phuong Le )

${ }^{1}$ Present address: 208 S Arnaz Dr., Apt. A, Beverly Hills, CA 90211.
} 
to pay the externality that he imposes on other bidders. I propose two modifications of the VCG mechanism, truncation $V C G$ and budget $V C G$, that circumvent this issue while attaining desirable incentive and efficiency properties.

Truncation VCG first truncates each bidder's valuations at his budget and then applies the VCG mechanism to the resulting truncated valuations. Because truncation VCG discards the variation of valuations above budgets, it is neither Pareto-Optimal nor incentive compatible in general. However, when bidders are single-minded, this loss of information is minimal and truncation VCG is individually rational, incentive compatible and Pareto-Optimal. Truncation VCG can therefore be suitable for applications where the single-minded assumption is appropriate, such as auctioning of pollution rights, communication links in a tree or auto parts to buyers desiring a specific model (see Lehmann et al., 2002, and the references therein). Other potential applications are spectrum auctions where the auctioneer has sufficient information about a bidder's existing technology and wireless infrastructures to be confident that the bidder is only interested in only one specific spectrum bundle.

Budget VCG uses the idea that a bidder must be able to pay the externality that he imposes on other bidders, so only allocations that result in affordable externalities can be chosen. This set of allocations is called the affordable set. Intersecting all affordable sets results in a set of allocations that are jointly affordable by all bidders. The surplus-maximizing allocation from this set is then chosen, and payments are the corresponding externalities which are, by construction, affordable to the winners. Budget VCG induces bidders to reveal their budgets truthfully and, when a non-null outcome is realized, attains ParetoOptimality. Because budget VCG may result in large inefficiencies when the null outcome is realized, it is only appropriate in settings where budget constraints are not too restrictive.

\section{Setting}

A seller $S$ wants to allocate a set $G$ of indivisible goods to a set $I$ of bidders. Let $X$ be the set of feasible allocations $x$, where $x=\left(x_{1}, x_{2}, \ldots, x_{I}\right)$ specifies that bidder $i$ gets bundle $x_{i}$ and must satisfy $x_{i} \cap x_{j}=\emptyset$ for all $i \neq j$. A bidder $i$ 's valuations over the bundles are summarized by a function $u_{i}: 2^{G} \rightarrow \mathbb{R}^{+}$. The valuation from the empty bundle is zero. I assume each bidder only cares about his own bundle and write $u_{i}(x)$ to mean $u_{i}\left(x_{i}\right)$. A bidder $i$ is single-minded if there is a bundle $\bar{x}_{i}$ such that for any bundle $x_{i}, u_{i}\left(x_{i}\right)=u_{i}\left(\bar{x}_{i}\right)$ if $x_{i} \supseteq \bar{x}_{i}$ and $u_{i}\left(x_{i}\right)=0$ otherwise. In the single-minded domain, all bidders are single-minded. A bidder $i$ also has a budget $b_{i} \in \mathbb{R}^{+}$that limits how much he can afford to pay. A bidder's valuation function and budget are private information, unknown to other bidders and the seller. A profile $(u, b)=\left(u_{i}, b_{i}\right)_{i \in I}$ describes the characteristics of all bidders. Let $U \times B$ denote the set of profiles.

Let $P=\left\{p: p=\left(p_{i}\right)_{i \in I} \in \mathbb{R}^{I}\right\}$ be the set of payments. An outcome is a pair $(x, p) \in X \times P$ that specifies that bidder $i$ gets bundle $x_{i}$ and pays $p_{i}$. Given an outcome $(x, p)$, the payoff for bidder $i$ is given by $v_{i}(x, p)=u_{i}(x)-p_{i}$ if $p_{i} \leq b_{i}$ and $-\infty$ otherwise. The seller's valuation is assumed to be identically zero. The payoff for the seller is the total payment $v_{S}(x, p)=\sum_{i \in I} p_{i}$.

A direct mechanism elicits valuations and budgets from the bidders and then maps each profile to an outcome using a function $\phi: U \times B \rightarrow X \times P$. A mechanism is individually rational if each bidder's payoff is non-negative. A mechanism is incentive compatible if it is each bidder's dominant strategy to report his valuations and budgets truthfully in the elicitation stage of the mechanism. The outcome $(x, p)$ is ParetoOptimal at profile $(u, b)$ if there is no outcome $(y, q)$ such that $v_{i}(y, q) \geq v_{i}(x, p)$ for all $i \in I+S$, with strict inequality for some $i \in I+S$. Note that in the presence of budget constraints, a Pareto-Optimal outcome need not involve the surplus-maximizing allocation. A mechanism is Pareto-Optimal if its outcome is always Pareto-Optimal at the input profile. 


\section{Results}

\subsection{Truncation VCG}

Restrict attention to the single-minded domain. A bidder $i$ 's truncated valuation is a function $u_{i}^{b}: 2^{N} \rightarrow$ $\mathbb{R}^{+}$such that for any bundle $x_{i}, u_{i}^{b}\left(x_{i}\right)=\min \left\{u_{i}\left(x_{i}\right), b_{i}\right\}$. Let $V_{C}^{b}(x)=\sum_{i \in C} u_{i}^{b}(x)$ denote total truncated valuation of bidders in group $C$ at allocation $x$, and let $V_{C}^{b}=\max _{x \in X} V_{C}^{b}(x)$ be the maximized truncated valuation for the same group. Truncation VCG chooses an allocation that maximizes total truncated valuation, $x^{V C G}(u)=\arg \max _{x \in X} V_{I}^{b}(x)$, and charges the associated externalities, $p_{i}^{V C G}(u)=V_{I \backslash i}^{b}-V_{I \backslash i}^{b}\left(x^{V C G}(u)\right)$.

A profile is generic if truncation VCG yields an unique allocation. ${ }^{2}$

Theorem 1. Suppose all bidders are single-minded. Then truncation VCG is individually rational and Pareto-Optimal. Moreover, at generic profiles, truthful reporting of valuations and budgets is optimal for each bidder.

Proof. Individual rationality is obvious. For Pareto-Optimality, suppose that there is an outcome $(y, q)$ that Pareto dominates a truncation VCG outcome $(x, p)$. Let $W$ be the set of bidders winning at both outcomes, $W_{x}$ be the set of bidders winning at $x$ but losing at $y$, and $W_{y}$ be the set of bidders losing at $x$ but winning at $y$. Because truncation VCG maximizes truncated valuation, I have $V_{W}^{b}(x)+V_{W_{x}}^{b}(x)+V_{W_{y}}^{b}(x) \geq$ $V_{W}^{b}(y)+V_{W_{x}}^{b}(y)+V_{W_{y}}^{b}(y)$. By definition and single-mindedness, $u_{i}^{b}(x)=u_{i}^{b}(y)$ for all $i \in W, V_{W_{x}}^{b}(y)=0$ and $V_{W_{y}}^{b}(x)=0$, so the previous inequality implies $V_{W_{x}}^{b}(x) \geq V_{W_{y}}^{b}(y)$.

For bidders in $W$, Pareto dominance and single-mindedness imply they must not be paying more at $(y, q)$ than at $(x, p)$, i.e., $q_{i} \leq p_{i}$ for all $i \in W$, so seller's revenue from $W$ at $(y, q)$ does not exceed that at $(x, p)$. At $(x, p)$, bidders in $W_{x}$ get a total payoff of at least $V_{W_{x}}^{b}(x)-\sum_{i \in W_{x}} p_{i}$, and the seller gets $\sum_{i \in W_{x}} p_{i}$ from them. Since $(y, q)$ Pareto dominates $(x, p)$, the bidders in $W_{y}$ must pay the bidders in $W_{x}$ a total of at least $V_{W_{x}}^{b}(x)-\sum_{i \in W_{x}} p_{i}$ and pay the seller at least $\sum_{i \in W_{x}} p_{i}$, for a total payment of $V_{W_{x}}^{b}(x)$. Thus, it must be that $V_{W_{y}}^{b}(y) \geq V_{W_{x}}^{b}(x)$. Pareto dominance requires that at least a bidder or the seller is strictly better off, so the inequality must be strict, i.e., $V_{W_{y}}^{b}(y)>V_{W_{x}}^{b}(x)$, contradicting the last inequality from the previous paragraph.

For optimality of truthful reporting, consider any generic profile $(u, b)$ and any bidder $i$ at allocation $x^{V C G}\left(u^{b}\right)$. Suppose $i$ is losing, then his payoff must then be zero, and $V_{I}^{b}=V_{I-i}^{b}$. By genericity, for any alternative allocation $y, V_{I}^{b}(y)<V_{I}^{b}=V_{I-i}^{b}$, which implies $V_{I-i}^{b}-V_{I \backslash i}^{b}(y)>u_{i}^{b}(y)$. This means that any misreport that attains $y$ is not profitable for $i$ because he would have to pay $V_{I-i}^{b}-V_{I \backslash i}^{b}(y)>u_{i}^{b}(y)$.

Now suppose $i$ is winning his bundle of interest $\bar{x}_{i}$ at the truncation VCG allocation $x^{V C G}$. Any allocation $y$ with $y_{i} \nsupseteq \bar{x}_{i}$ gives $i$ valuation of zero, so $i$ has no incentive to try to realize it. Consider any allocation $y$ with $y_{i} \supseteq \bar{x}_{i}$. By genericity and the fact that $x^{V C G}$ maximizes truncated valuation, $V_{I \backslash i}^{b}\left(x^{V C G}\right)+u_{i}^{b}\left(x^{V C G}\right)=V_{I}^{b}>$ $V_{I}^{b}(y)=V_{I \backslash i}^{b}(y)+u_{i}^{b}(y)$. Noting that $u_{i}^{b}\left(x^{V C G}\right)=u_{i}^{b}(y)$ yields $V_{I \backslash i}^{b}\left(x^{V C G}\right)>V_{I \backslash i}^{b}(y)$. Hence, $V_{I \backslash i}-V_{I \backslash i}^{b}(y)>$ $V_{I \backslash i}-V_{I \backslash i}^{b}\left(x^{V C G}\right)$. Therefore, bidder $i$ will pay more for $y_{i}$ than for $\bar{x}_{i}$ if he misreports to realize allocation $y$. Since both $y_{i}$ and $\bar{x}_{i}$ yield the same valuation, such a misreport is unprofitable.

\subsection{Budget $V C G$}

Let $V_{C}(x)=\sum_{i \in C} u_{i}(x)$ denote the total valuation of bidders in $C$ at allocation $x$. Let $V_{C}=\max _{x \in X} V_{C}(x)$ denote the maximized total valuation of coalition $C$. Let $F_{i}(u, b)=\left\{x: V_{I \backslash i}-V_{I \backslash i}(x) \leq b^{i}\right\}$ be the set of allocations affordable by bidder $i$. Let $F(u, b)=\cap_{i \in I} F_{i}(u, b)$ be the set of allocations affordable by all

\footnotetext{
${ }^{2}$ Genericity can be relaxed to require only that truncation VCG yields an unique payoff profile and theorem 1 will still hold.
} 
bidders. If $F(u, b)$ is non-empty, budget VCG chooses the valuation-maximizing allocation from $F(u, b)$, $x^{*}=\arg \max _{x \in F(u, b)} V_{I}(x)$. Payments are externalities: $p_{i}^{*}=V_{I \backslash i}-V_{I \backslash i}\left(x^{*}\right)$. If $F(u, b)$ is empty, budget VCG sets $x_{i}^{*}=$ and $p_{i}^{*}=0$ for all $i$.

Theorem 2. Budget VCG is individually rational, and, when it implements a non-null allocation, ParetoOptimal. Furthermore, it is a dominant strategy for the bidders to report their budgets truthfully.

Proof. Individual rationality is obvious. For Pareto-Optimality, suppose a non-null outcome $\left(x^{*}, p^{*}\right)$ is chosen. Note that at $x^{*}$, total payoff is $V_{I}\left(x^{*}\right)$, bidder $i$ gets payoff $V_{I}\left(x^{*}\right)-V_{I \backslash i}$, so the total payoff of bidders in $(I \backslash i)$ together with the seller is $V_{I \backslash i}$. Suppose that another outcome $(y, q)$ Pareto dominates $\left(x^{*}, p^{*}\right)$. Then it must be that $V_{I}(y)>V_{I}\left(x^{*}\right)$. This implies that $y \notin F(u, b)$, so $y \notin F_{i}(u, b)$ for some $i$. By definition, this means that $V_{I \backslash i}-V_{I \backslash i}(y)>b_{i}$. Rearranging yields $V_{I \backslash i}>V_{I \backslash i}(y)+b_{i}$. The right hand side of the inequality is the maximum total payoff that bidders $I-i$ and the seller can get at allocation $y$ : it is the sum of the total valuation of coalition $I \backslash i$ at $y$ and the maximum payment from $i$. The left hand side is the total payoff that the same bidders and seller get at outcome $\left(x^{*}, p^{*}\right)$. Therefore at least one agent must be strictly worse off at $(y, q)$, contradicting Pareto dominance.

For optimality of truthful reporting of budget, when a non-null outcome is chosen, payoff for bidder $i$ is $v_{i}=V_{I}\left(x^{*}\right)-V_{I \backslash i}=\max _{x \in F(u, b)} V_{I}(x)-V_{I \backslash i}$. The second term is independent of bidder $i$ 's report so any profitable budget misreport must raise the first term. Suppose that such a profitable misreport $\hat{b}_{i}$ exists and results in the allocation $\hat{x}$, then it must be that $\hat{x} \in F\left(u, \hat{b}_{i}, b_{-i}\right)$ but $\hat{x} \notin F(u, b)$ because $V_{I}(\hat{x})>\max _{x \in F(u, b)} V_{I}(x)$.

Recall that $F_{i}(u, b)=\left\{x: V_{I \backslash i}-V_{I \backslash i}(x) \leq b^{i}\right\}$ and $F(u, b)=\cap_{i \in I} F_{i}(u, b)$. So $\hat{x} \in F\left(u, \hat{b}_{i}, b_{-i}\right)$ implies that $\hat{x} \in F_{j}\left(u, \hat{b}_{i}, b_{-i}\right)$ for all $j$. Because $j$ ' affordable set does not depend on $i$ 's budget, $F_{j}\left(u, \hat{b}_{i}, b_{-i}\right)=F_{j}(u, b)$ for all $j \neq i$. Hence, $\hat{x} \in F_{j}(u, b)$ for all $j \neq i$. Because $\hat{x} \notin F(u, b)$, it must be that $\hat{x} \notin F_{i}(u, b)$. Therefore, $i$ 's payment at $\hat{x}$ exceeds his true budget $b_{i}$, so $\hat{x}$ cannot yield a higher payoff for $i$. A similar logic works for the case where a null outcome is chosen.

Acknowledgements I am grateful for comments and suggestions from participants at the Econ Theory Seminar at Stanford University.

\section{References}

Ausubel, L. M. (2004). An efficient ascending-bid auction for multiple objects. American Economic Review, 94(5):1452-1475.

Bhattacharya, S., Conitze, V., Munagala, K., and Xia, L. (2010). Incentive compatible budget elicitation in multi-unit auctions. In In Proceedings of the Annual AM-SIAM Symposium on Disrete Algorithms, pages 554-572.

Borgs, C., Chayes, J., Immorlica, N., Mahdian, M., and Saberi, A. (2005). Multi-unit auctions with budget-constrained bidders. In EC 05: Proceedings of the 6th ACM conference on Electronic commerce, pages 44-51.

Bulow, J., Levin, J., and Milgrom, P. (2009). Winning play in spectrum auctions. Technical report, National Bureau of Economic Research.

Che, Y.-K. and Gale, I. (1996). Expected revenue of all-pay auctions and first-price sealed-bid auctions with budget constraints. Economic Letters, 50:373-379.

Che, Y.-K. and Gale, I. (2000). The optimal mechanism for selling to a budget-constrained buyer. Journal of Economic Theory, 92:198-2000.

Dobzinski, S., Lavi, R., and Nisan, N. (2008). Multi-unit auctions with budget limits. FOCS.

Fiat, A., Leonardi, S., Saia, J., and Sankowski, P. (2011). Single valued combinatorial auctions with budgets. ACM Conference on Electronic Commerce.

Goel, G., Mirrokni, V., and Leme, R. P. (2012). Polyhedral clinching auctions and the adwords polytope. In STOC '12 Proceedings of the forty-fourth annual ACM symposium on the Theory of Computing, pages 107-122. ACM New York.

Gul, F. and Stacchetti, E. (2000). The English auction with differentiated commodities. Journal of Economic theory, 92(1):66-95.

Hafalir, I. E., Ravi, R., and Sayedi, A. (2011). A near Pareto-optimal auction with budget constraints. Games and Economic Behavior, 74:699-708. 
Laffront, J.-J. and Robert, J. (1996). Optimal auction with financially constrained buyers. Economic Letters, 52(2):181-186.

Lavi, R. and May, M. (2011). A note on the incompatibility of strategy-proofness and pareto-optimality in quasi-linear settings with public budgets. In Internet and Network Economics, Lecture Notes in Computer Science, page 417. Springer Berlin Heidelberg.

Lehmann, D., Oćallaghan, L. I., and Shoham, Y. (2002). Truth revelation in approximately efficient combinatorial auctions. J. ACM, 49(5):577-602.

Milgrom, P. (2000). Putting auction theory to work: The simultaneous ascending auction. Journal of Political Economy, 108(2):245-272. 